\title{
Chemical Composition of Abaca (Musa textilis) Leaf Fibers Used for Manufacturing of High Quality Paper Pulps
}

\author{
José C. DEL Río* AND ANA GutiÉRREZ \\ Instituto de Recursos Naturales y Agrobiología de Sevilla, CSIC, P.O. Box 1052, \\ E-41080 Seville, Spain
}

\begin{abstract}
The chemical composition of leaf fibers of abaca (Musa textilis), which are commonly used for highquality paper pulp production, was thoroughly studied. The results revealed that the lignin content was $13.2 \%$ of the total fiber. The analysis of abaca fibers by pyrolysis coupled to gas chromatographymass spectrometry (Py-GC/MS) released predominantly compounds arising from lignin and $p$ hydroxycinnamic acids, with high amounts of 4-vinylphenol. The latter compound was demonstrated to arise from $p$-coumaric acid by pyrolysis of abaca fibers in the presence of tetramethylammonium hydroxide, which released high amounts of $p$-coumaric acid (as the methyl derivative). Products from p-hydroxyphenyl $(\mathrm{H})$, guaiacyl $(\mathrm{G})$, and syringyl $(\mathrm{S})$ propanoid units, with a predominance of the latter (H:G:S molar ratio of 1.5:1:4.9), were also released after Py-GC/MS of abaca fibers. Sinapyl and coniferyl acetates, which are thought to be lignin monomer precursors, were also found in abaca. The extractives content of the abaca fiber $(0.4 \%)$ was low, and the most predominant compounds were free sterols ( $24 \%$ of total extract) and fatty acids ( $24 \%$ of total extract). Additionally, significant amounts of steroid ketones (10\%), triglycerides $(6 \%), \omega$-hydroxyfatty acids $(6 \%)$, monoglycerides $(4 \%)$, fatty alcohols ( $4 \%)$, and a series of $p$-hydroxycinnamyl ( $p$-coumaric and ferulic acids) esterified with long chain alcohols and $\omega$-hydroxyfatty acids were also found, together with minor amounts of steroid hydrocarbons, diglycerides, $\alpha$-hydroxyfatty acids, sterol esters, and sterol glycosides.
\end{abstract}

KEYWORDS: Abaca; Musa textilis; leaf fibers; nonwood; pulps; lipids; sterols; lignin; pyrolysis

\section{INTRODUCTION}

Nonwood fibers have a long history as papermaking raw materials. Although wood is still by far the main raw material for paper pulp manufacturing in developed countries, a market exists for high value-added papers from nonwood fibers. Indeed, their prices are higher as compared to wood kraft pulp. Moreover, where wood-based fibers are not available, as in the developing world, nonwood plants are the dominant fiber source for papermaking. Beside cereal straw, the leading nonwoody fiber and one of the oldest sources of paper pulp, several crops are grown for their content in long fibers. Flax, hemp, abaca, kenaf, jute, and sisal are among the nonwood fibers used in the manufacturing of high-quality pulps for specialty papers (such as tea bags, baby napkins, machine filters, bank notes, security papers, cigarette papers, or condenser papers).

Among the nonwoody fibers, the leaf fibers of abaca (Musa textilis), an annual plant from the Musaceae family similar to the banana tree, are an excellent raw material for manufacturing specialty papers. Its long fiber length, high strength, and fineness make it a superior material for the production of thin, lightweight papers of high porosity and excellent tear, burst, and tensile indices (1). There have been several studies on abaca pulping

* To whom correspondence should be addressed. Tel: +34-95-4624711. Fax: +34-95-4624002. E-mail: delrio@irnase.csic.es. and bleaching (2-7). However, despite this abundant literature on abaca pulping, there is a lack of papers describing the chemical composition of this fiber. Besides the previous papers reporting the characterization of polysaccharides and lignin $(8$, 9) and our previous work describing the identification of $p$-hydroxycinnamyl esters in abaca fiber (10), there have not been more published studies on the chemical composition of this interesting raw material. Studies on the chemical composition of abaca fibers are important for optimizing the pulping and bleaching processes of this raw material. The composition of lignin and lipids in fibers can strongly influence pulping and bleaching processes. Thus, it is known that the efficiency of pulping is directly proportional to the amount of syringyl (S) units in lignin $(11,12)$. On the other hand, it is well-known that lipophilic compounds present in raw materials cause significant environmental and technical problems in the manufacturing of paper pulp. During pulping, lipids are released from the fibers forming colloidal pitch, which can deposit in either pulp or machinery causing production troubles $(13-15)$. In the manufacture of alkaline pulps, a large part of the lipids originally present in the raw material is removed during the cooking. However, some chemical species survive these processes and are found as pulp extractives, suspended in process waters or 


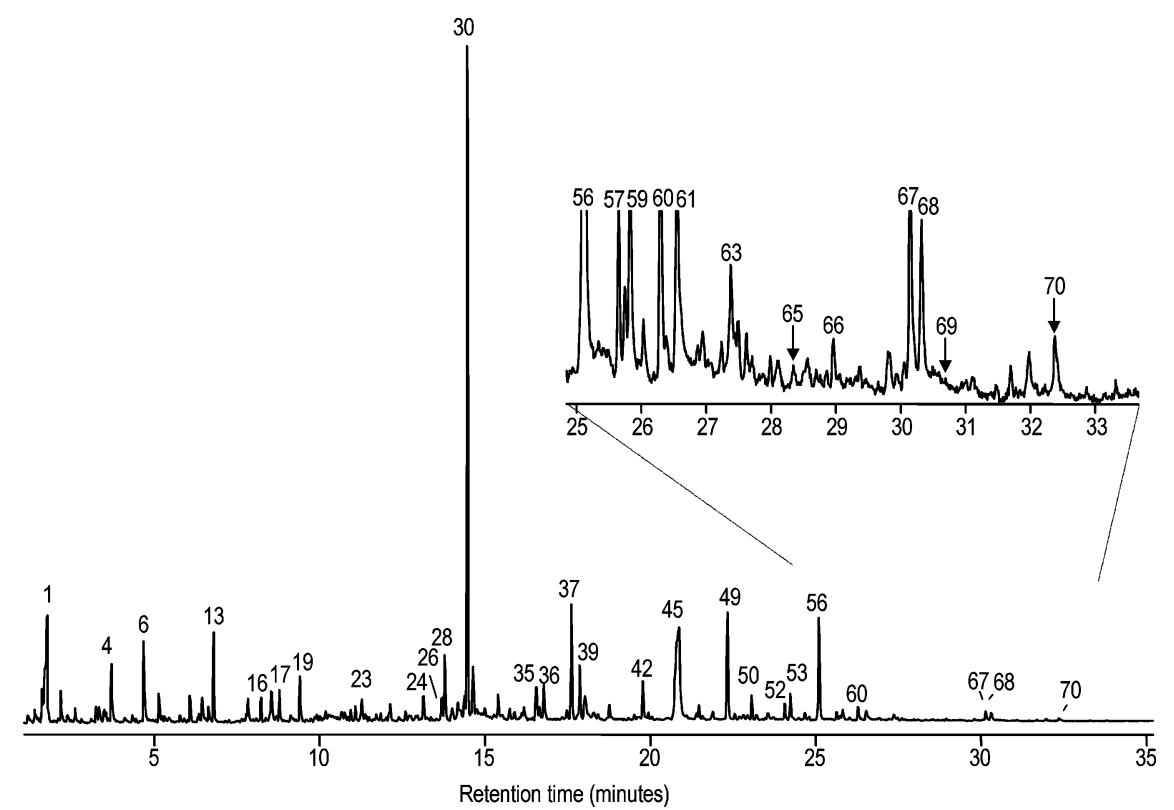

Figure 1. Py-GC/MS chromatogram of abaca fibers. The identities and relative molar abundances of the compounds are listed in Table 2.

Table 1. Composition (ppm) of the Different Metals in the Abaca Fibers

\begin{tabular}{crcccccc}
\hline elements & abaca & elements & abaca & elements & abaca & elements & abaca \\
\hline $\mathrm{K}$ & 1735 & $\mathrm{Fe}$ & 24 & $\mathrm{Si}$ & 6 & $\mathrm{~B}$ & 2 \\
$\mathrm{Ca}$ & 992 & $\mathrm{Mn}$ & 18 & $\mathrm{Zn}$ & 4 & $\mathrm{Cu}$ & 1 \\
$\mathrm{Na}$ & 75 & $\mathrm{Al}$ & 15 & $\mathrm{Ba}$ & 3 & $\mathrm{Ni}$ & 1 \\
$\mathrm{Mg}$ & 65 & $\mathrm{Sr}$ & 10 & $\mathrm{Cr}$ & 2 & $\mathrm{~Pb}$ & 1 \\
\hline
\end{tabular}

forming the so-called pitch deposits in circuits, equipment, and the final product $(13-15)$. Moreover, such extractives might contribute to the toxicity of paper pulp effluents and products $(16,17)$. In the present study, we report the chemical composition of the leaf fibers of abaca used in paper pulp manufacture with special emphasis on the lignin and lipophilic compounds.

\section{MATERIALS AND METHODS}

Sample. Abaca (M. textilis) leaf fibers were supplied by CELESA mill (Tortosa, Spain). Abaca fibers were air-dried, milled using a knife mill (Janke and Kunkel, Analyzenmühle), and successively extracted with acetone in a Soxhlet apparatus for $8 \mathrm{~h}$ and with hot water $(3 \mathrm{~h}$ at $\left.100{ }^{\circ} \mathrm{C}\right)$. The acetone extracts were evaporated to dryness and resuspended in chloroform for chromatographic analysis of the lipophilic fraction. The Klason lignin content was estimated as the residue after sulfuric acid hydrolysis of the pre-extracted material, and neutral sugars from carbohydrate hydrolysis were analyzed as alditol acetates by gas chromatography (GC) according to Tappi rules T222 om-88 and T249 om85, respectively (18). The ash content was estimated as the residue after $6 \mathrm{~h}$ at $575{ }^{\circ} \mathrm{C}$. Two replicates were used for each sample. The composition (as percent of whole fiber) was as follows: ash, $0.5 \%$; acetone extractives, $0.4 \%$; water soluble extracts, $0.3 \%$; Klason lignin, $11.8 \%$; and acid soluble lignin, $1.4 \%$. The composition of neutral monosaccharides after acid hydrolysis (as percent of total neutral carbohydrates) included rhamnose, $0.2 \%$; arabinose, $1.6 \%$; xylose, $7.5 \%$; mannose, $3,5 \%$; galactose, $0.2 \%$; and glucose, $87 \%$. No uronic acid determination was performed in this study.

Inductively Coupled Plasma Spectrophotometry (ICP-OES). The different metals were extracted by wet oxidation with concentrated $\mathrm{HNO}_{3}$ under pressure in a microwave digester. Analysis of the mineral nutrients in the extracts thus obtained was performed by ICP-OES. The accuracy of the analytical method was assessed by carrying out analyses of three BCR (Community Bureau of Reference) reference samples: BCR 62 (Olive leaves), CRM 279 (Sea lettuce), and CRM 281 (Ryegrass) (19-21)
Fractionation by Solid Phase Extraction (SPE). The lipid extracts were fractionated by a SPE procedure developed by Gutiérrez et al. (22) using aminopropyl phase cartridges $(500 \mathrm{mg}$ ) from Waters (Division of Millipore). The dried chloroform extracts were taken up in a minimal volume $(<0.5 \mathrm{~mL})$ of hexane:chloroform $(4: 1)$ and loaded into the cartridge column previously conditioned with hexane $(4 \mathrm{~mL})$. The cartridge was loaded and eluted by gravity. The column was first eluted with $8 \mathrm{~mL}$ of hexane (fraction A) and subsequently with $6 \mathrm{~mL}$ of hexane:chloroform (5:1) (fraction B), then with $10 \mathrm{~mL}$ of chloroform (fraction C) and finally with $10 \mathrm{~mL}$ of diethyl ether:acetic acid (98:2) (fraction D). Each isolated fraction was dried under nitrogen and analyzed by GC and GC/MS.

Saponification of Sterol Esters. The SPE fraction enriched in sterol esters was hydrolyzed by refluxing it with a $0.5 \mathrm{M}$ solution of potassium hydroxide in $90 \%$ ethanol for $8 \mathrm{~h}$. The solution was thoroughly extracted with hexane and dried over anhydrous sodium sulfate, and the nonsaponifiable materials were recovered on removal of the solvent in a rotary evaporator. The aqueous layer was acidified with $6 \mathrm{M}$ hydrochloric acid and extracted with hexane. The free fatty acids were recovered after drying the extract over anhydrous sodium sulfate and removing the solvent in the usual way. Both the neutral and the acidic fractions were analyzed by GC and GC/MS.

GC and GC/MS Analyses. An HP 5890 gas chromatograph (Hewlett-Packard, Hoofddorp, Netherlands) equipped with a splitsplitless injector and a flame ionization detector (FID) was used for $\mathrm{GC}$ analyses. The injector and the detector temperatures were set at 300 and $350{ }^{\circ} \mathrm{C}$, respectively. Samples were injected in the splitless mode. Helium was used as the carrier gas. The capillary column used was a high temperature, polyimide-coated fused silica tubing DB-5HT $(5 \mathrm{~m} \times 0.25 \mathrm{~mm}$ i.d., $0.1 \mu \mathrm{m}$ film thickness) from $\mathrm{J} \& \mathrm{~W}$ Scientific (Folsom, CA), especially processed for use at $400{ }^{\circ} \mathrm{C}$. The oven was temperature-programmed from $100(1 \mathrm{~min})$ to $350^{\circ} \mathrm{C}(3 \mathrm{~min})$ at $15^{\circ} \mathrm{C}$ $\min ^{-1}$. Peaks were quantified by area, and a mixture of standards (octadecane, palmitic acid, sitosterol, cholesteryl oleate, campesteryl, stigmasteryl, and sitosteryl 3- $\beta$-D-glucopyranosides) was used to elaborate calibration curves. The data from the two replicates were averaged. In all cases, the standard deviations from replicates were below $10 \%$ of the mean values.

The GC/MS analyses were performed on a Varian Saturn 2000 (Varian, Walnut Creek, CA) with an ion trap detector, equipped with a fused silica capillary column (DB-5HT, J\&W; $15 \mathrm{~m} \times 0.25 \mathrm{~mm}$ i.d., $0.1 \mu \mathrm{m}$ film thickness). The transfer line was kept at $300{ }^{\circ} \mathrm{C}$. The injector was temperature programmed from $120^{\circ} \mathrm{C}(0.1 \mathrm{~min})$ to 380 ${ }^{\circ} \mathrm{C}$ at a rate of $200{ }^{\circ} \mathrm{C} / \mathrm{min}$ and held until the end of the analysis. The injector and transfer line temperatures were set at 300 and $350{ }^{\circ} \mathrm{C}$, respectively. Helium was used as the carrier gas, and the injection was 
Table 2. Composition and Relative Molar Abundances (\%) of the Compounds Released after Py-GC/MS of Abaca (M. textilis) Fibers ${ }^{a}$

\begin{tabular}{|c|c|c|c|c|c|c|}
\hline no. & compounds & mass fragments & MW & formula & origin & $\%$ \\
\hline 1 & hydroxyacetaldehyde & $42 / 60$ & 60 & $\mathrm{C}_{2} \mathrm{H}_{4} \mathrm{O}_{2}$ & C & 15.1 \\
\hline 2 & 3-hydroxypropanal & $73 / 74$ & 74 & $\mathrm{C}_{3} \mathrm{H}_{6} \mathrm{O}_{2}$ & $\mathrm{C}$ & 1.4 \\
\hline 3 & $(3 \mathrm{H})$-furan-2-one & $55 / 84$ & 84 & $\mathrm{C}_{4} \mathrm{H}_{4} \mathrm{O}_{2}$ & C & 0.7 \\
\hline 4 & 2,3-butanedione & $56 / 57 / 86$ & 86 & $\mathrm{C}_{4} \mathrm{H}_{6} \mathrm{O}_{2}$ & C & 3.1 \\
\hline 5 & $(2 H)$-furan-3-one & $55 / 84$ & 84 & $\mathrm{C}_{4} \mathrm{H}_{4} \mathrm{O}_{2}$ & C & 0.5 \\
\hline 6 & 2-furaldehyde & $67 / 95 / 96$ & 96 & $\mathrm{C}_{5} \mathrm{H}_{4} \mathrm{O}_{2}$ & C & 3.9 \\
\hline 7 & 2-methylfuran & $53 / 81 / 82$ & 82 & $\mathrm{C}_{5} \mathrm{H}_{6} \mathrm{O}$ & C & 0.3 \\
\hline 8 & 2-(hydroxymethyl)furan & $43 / 70 / 81 / 98$ & 98 & $\mathrm{C}_{5} \mathrm{H}_{6} \mathrm{O}_{2}$ & C & 1.3 \\
\hline 9 & cyclopent-1-ene-3,4-dione & $54 / 68 / 96$ & 96 & $\mathrm{C}_{5} \mathrm{H}_{4} \mathrm{O}_{2}$ & C & 0.3 \\
\hline 10 & 4-methyltetrahydrofuran-3-one & $43 / 72$ & 100 & $\mathrm{C}_{5} \mathrm{H}_{8} \mathrm{O}_{2}$ & C & 0.9 \\
\hline 11 & $(5 H)$-furan-2-one & $55 / 84$ & 84 & $\mathrm{C}_{4} \mathrm{H}_{4} \mathrm{O}_{2}$ & C & 1.4 \\
\hline 12 & acetylfuran & $43 / 95 / 110$ & 110 & $\mathrm{C}_{6} \mathrm{H}_{6} \mathrm{O}_{2}$ & C & 0.1 \\
\hline 13 & 2,3-dihydro-5-methylfuran-2-one & $55 / 69 / 98$ & 98 & $\mathrm{C}_{5} \mathrm{H}_{6} \mathrm{O}_{2}$ & C & 3.6 \\
\hline 14 & 5-methyl-2-furfuraldehyde & $53 / 109 / 110$ & 110 & $\mathrm{C}_{6} \mathrm{H}_{6} \mathrm{O}_{2}$ & C & 0.3 \\
\hline 15 & phenol & $65 / 66 / 94$ & 94 & $\mathrm{C}_{5} \mathrm{H}_{6} \mathrm{O}$ & $\mathrm{LH}$ & 0.9 \\
\hline 16 & 5,6-dihydropyran-2,5-dione & $68 / 98$ & 98 & $\mathrm{C}_{5} \mathrm{H}_{6} \mathrm{O}_{2}$ & C & 1.7 \\
\hline 17 & 4-hydroxy-5,6-dihydro-(2H)-pyran-2-one & $58 / 85 / 114$ & 114 & $\mathrm{C}_{5} \mathrm{H}_{6} \mathrm{O}_{3}$ & $\mathrm{C}$ & 1.1 \\
\hline 18 & 3-hydroxy-2-methyl-2-cyclopenten-1-one & $55 / 84 / 112$ & 112 & $\mathrm{C}_{6} \mathrm{H}_{8} \mathrm{O}_{2}$ & C & 0.4 \\
\hline 19 & 2-hydroxy-3-methyl-2-cyclopenten-1-one & $55 / 84 / 112$ & 112 & $\mathrm{C}_{6} \mathrm{H}_{8} \mathrm{O}_{2}$ & C & 1.8 \\
\hline 20 & 2,3-dimethylcyclopenten-1-one & $67,95,110$ & 110 & $\mathrm{C}_{7} \mathrm{H}_{10} \mathrm{O}$ & C & 0.1 \\
\hline 21 & 4-methylphenol & $77 / 107 / 108$ & 108 & $\mathrm{C}_{7} \mathrm{H}_{8} \mathrm{O}$ & $\mathrm{LH}$ & 0.4 \\
\hline 22 & 2-furoic acid, methyl ester & $67 / 95 / 126$ & 126 & $\mathrm{C}_{6} \mathrm{H}_{6} \mathrm{O}_{3}$ & $\mathrm{C}$ & 0.3 \\
\hline 23 & guaiacol & $81 / 109 / 124$ & 124 & $\mathrm{C}_{7} \mathrm{H}_{8} \mathrm{O}_{2}$ & LG & 0.5 \\
\hline 24 & 4-ethylphenol & $77 / 107 / 122$ & 122 & $\mathrm{C}_{8} \mathrm{H}_{10} \mathrm{O}_{2}$ & $\mathrm{LH}$ & 0.7 \\
\hline 25 & 3,4-dihydroxybenzaldehyde & $81 / 109 / 137 / 138$ & 138 & $\mathrm{C}_{7} \mathrm{H}_{6} \mathrm{O}_{3}$ & $\mathrm{~L}$ & 0.3 \\
\hline 26 & 5-hydroxymethyl-2-tetrahydrofuraldehyde-3-one & $43 / 57 / 69 / 70 / 85$ & 144 & $\mathrm{C}_{6} \mathrm{H}_{8} \mathrm{O}_{4}$ & C & 0.7 \\
\hline 27 & 4-methylguaiacol & $95 / 123 / 138$ & 138 & $\mathrm{C}_{8} \mathrm{H}_{10} \mathrm{O}_{2}$ & LG & 0.1 \\
\hline 28 & catechol & $64 / 81 / 92 / 110$ & 110 & $\mathrm{C}_{6} \mathrm{H}_{6} \mathrm{O}_{2}$ & $\mathrm{~L} / \mathrm{C}$ & 2.3 \\
\hline 29 & 5-hydroxymethyl-2-furaldehyde isomer & $69 / 97 / 109 / 126$ & 126 & $\mathrm{C}_{6} \mathrm{H}_{6} \mathrm{O}_{3}$ & $\mathrm{C}$ & 0.7 \\
\hline 30 & 4-vinylphenol & $65,91,120$ & 120 & $\mathrm{C}_{8} \mathrm{H}_{8} \mathrm{O}$ & $p \mathrm{Ca}$ & 23.0 \\
\hline 31 & 5-hydroxymethyl-2-furaldehyde & $69 / 97 / 109 / 126$ & 126 & $\mathrm{C}_{6} \mathrm{H}_{6} \mathrm{O}_{3}$ & $\mathrm{C}$ & 1.6 \\
\hline 32 & 3-methoxycatechol & $79 / 97 / 125 / 140$ & 140 & $\mathrm{C}_{7} \mathrm{H}_{8} \mathrm{O}_{3}$ & L & 0.7 \\
\hline 33 & 4-ethylguaiacol & $122 / 137 / 152$ & 152 & $\mathrm{C}_{9} \mathrm{H}_{12} \mathrm{O}_{2}$ & LG & 0.2 \\
\hline 34 & 4-methylcatechol & $78 / 107 / 123 / 124$ & 124 & $\mathrm{C}_{7} \mathrm{H}_{8} \mathrm{O}_{2}$ & L & 0.1 \\
\hline 35 & 1,4-dideoxy-D-glycerohex-1-enepyrenone-3-ulose & $43 / 73 / 87 / 113 / 144$ & 144 & $\mathrm{C}_{6} \mathrm{H}_{8} \mathrm{O}_{4}$ & C & 1.1 \\
\hline 36 & 4-vinylguaiacol & $107 / 135 / 150$ & 150 & $\mathrm{C}_{9} \mathrm{H}_{10} \mathrm{O}_{2}$ & $\mathrm{Fa} / \mathrm{LG}$ & 0.9 \\
\hline 37 & syringol & $111 / 139 / 154$ & 154 & $\mathrm{C}_{8} \mathrm{H}_{10} \mathrm{O}_{3}$ & LS & 3.3 \\
\hline 38 & eugenol & $131 / 149 / 164$ & 164 & $\mathrm{C}_{10} \mathrm{H}_{12} \mathrm{O}_{2}$ & LG & $<0.1$ \\
\hline 39 & 4-hydroxybenzaldehyde & $93,121,122$ & 122 & $\mathrm{C}_{7} \mathrm{H}_{6} \mathrm{O}_{2}$ & $\mathrm{LH}$ & 1.9 \\
\hline 40 & 1,2,3-benzenetriol & $52,80,108,126$ & 126 & $\mathrm{C}_{6} \mathrm{H}_{6} \mathrm{O}_{3}$ & $\mathrm{~L}$ & 1.9 \\
\hline 41 & vanillin & $109 / 151 / 152$ & 152 & $\mathrm{C}_{8} \mathrm{H}_{8} \mathrm{O}_{3}$ & LG & 0.3 \\
\hline 42 & 4-methylsyringol & $125 / 153 / 168$ & 168 & $\mathrm{C}_{9} \mathrm{H}_{12} \mathrm{O}_{2}$ & LS & 0.9 \\
\hline 43 & trans-isoeugenol & $131 / 149 / 164$ & 164 & $\mathrm{C}_{10} \mathrm{H}_{12} \mathrm{O}_{2}$ & LG & 0.1 \\
\hline 44 & homovanillin & $122 / 137 / 166$ & 166 & $\mathrm{C}_{10} \mathrm{H}_{14} \mathrm{O}_{2}$ & LG & 0.1 \\
\hline 45 & levoglucosane & $57 / 60 / 73 / 98$ & 162 & $\mathrm{C}_{6} \mathrm{H}_{10} \mathrm{O}_{5}$ & C & 9.6 \\
\hline 46 & 4-ethylsyringol & $167 / 182$ & 182 & $\mathrm{C}_{10} \mathrm{H}_{14} \mathrm{O}_{3}$ & LS & 0.3 \\
\hline 47 & guaiacylacetone & $122 / 137 / 180$ & 180 & $\mathrm{C}_{10} \mathrm{H}_{12} \mathrm{O}_{3}$ & LG & 0.1 \\
\hline 48 & 1,6-anhydro- $\beta$-D-glucofuranose & $73 / 85 / 115$ & 162 & $\mathrm{C}_{6} \mathrm{H}_{10} \mathrm{O}_{5}$ & $\mathrm{C}$ & 0.3 \\
\hline 49 & 4-vinylsyringol & $137 / 165 / 180$ & 180 & $\mathrm{C}_{10} \mathrm{H}_{12} \mathrm{O}_{3}$ & LS & 2.5 \\
\hline 50 & 4-allylsyringol & $167 / 179 / 194$ & 194 & $\mathrm{C}_{11} \mathrm{H}_{14} \mathrm{O}_{3}$ & LS & 0.6 \\
\hline 51 & 4-propylsyringol & $123 / 167 / 196$ & 196 & $\mathrm{C}_{11} \mathrm{H}_{16} \mathrm{O}_{3}$ & LS & 0.1 \\
\hline 52 & cis-4-propenylsyringol & $167 / 179 / 194$ & 194 & $\mathrm{C}_{11} \mathrm{H}_{14} \mathrm{O}_{3}$ & LS & 0.4 \\
\hline 53 & syringaldehyde & 167/181/182 & 182 & $\mathrm{C}_{9} \mathrm{H}_{10} \mathrm{O}_{4}$ & LS & 0.7 \\
\hline 54 & cis-coniferyl alcohol & $124 / 137 / 151 / 180$ & 180 & $\mathrm{C}_{10} \mathrm{H}_{12} \mathrm{O}_{3}$ & LG & $<0.1$ \\
\hline 55 & 4-propinylsyringol & 106/131/177/192 & 192 & $\mathrm{C}_{11} \mathrm{H}_{12} \mathrm{O}_{3}$ & LS & 0.2 \\
\hline 56 & trans-4-propenylsyringol & $167 / 179 / 194$ & 194 & $\mathrm{C}_{11} \mathrm{H}_{14} \mathrm{O}_{3}$ & LS & 2.4 \\
\hline 57 & acetosyringone & $153 / 181 / 196$ & 196 & $\mathrm{C}_{10} \mathrm{H}_{12} \mathrm{O}_{4}$ & LS & 0.2 \\
\hline 58 & trans-coniferaldehyde & $107 / 135 / 147 / 178$ & 178 & $\mathrm{C}_{10} \mathrm{H}_{10} \mathrm{O}_{3}$ & LG & 0.1 \\
\hline 59 & trans-coniferyl alcohol & $124 / 137 / 151 / 180$ & 180 & $\mathrm{C}_{10} \mathrm{H}_{12} \mathrm{O}_{3}$ & LG & 0.3 \\
\hline 60 & syringylacetone & $123 / 167 / 210$ & 210 & $\mathrm{C}_{11} \mathrm{H}_{14} \mathrm{O}_{4}$ & LS & 0.3 \\
\hline 61 & p-coumaric acid & $119,147,164$ & 164 & $\mathrm{C}_{9} \mathrm{H}_{8} \mathrm{O}_{3}$ & $\mathrm{pCa}$ & 0.4 \\
\hline 62 & cis-coniferyl acetate & $91,103,222$ & 222 & $\mathrm{C}_{12} \mathrm{H}_{14} \mathrm{O}_{2}$ & LG & $<0.1$ \\
\hline 63 & propiosyringone & $151 / 181 / 210$ & 210 & $\mathrm{C}_{11} \mathrm{H}_{14} \mathrm{O}_{4}$ & LS & 0.1 \\
\hline 64 & dihydrosinapyl alcohol & $167 / 168 / 212$ & 212 & $\mathrm{C}_{11} \mathrm{H}_{16} \mathrm{O}_{4}$ & LS & $<0.1$ \\
\hline 65 & trans-coniferyl acetate & $91,103,222$ & 222 & $\mathrm{C}_{12} \mathrm{H}_{14} \mathrm{O}_{2}$ & LG & $<0.1$ \\
\hline 66 & cis-sinapyl alcohol & $154 / 167 / 210$ & 210 & $\mathrm{C}_{11} \mathrm{H}_{14} \mathrm{O}_{4}$ & LS & $<0.1$ \\
\hline 67 & trans-sinapaldehyde & $137 / 165 / 180 / 208$ & 208 & $\mathrm{C}_{11} \mathrm{H}_{12} \mathrm{O}_{4}$ & LS & 0.2 \\
\hline 68 & trans-sinapyl alcohol & $154 / 167 / 210$ & 210 & $\mathrm{C}_{11} \mathrm{H}_{14} \mathrm{O}_{4}$ & LS & 0.2 \\
\hline 69 & cis-sinapyl acetate & $149 / 161 / 192 / 209 / 252$ & 252 & $\mathrm{C}_{13} \mathrm{H}_{16} \mathrm{O}_{5}$ & LS & $<0.1$ \\
\hline 70 & trans-sinapyl acetate & $149 / 161 / 192 / 209 / 252$ & 252 & $\mathrm{C}_{13} \mathrm{H}_{16} \mathrm{O}_{5}$ & LS & $<0.1$ \\
\hline
\end{tabular}

${ }^{a}$ Main mass fragments, molecular weight (MW), formula, and origin are included. C, cellulose; LH, p-hydroxycinnamyl lignin units; LG, guaiacyl lignin units; LS, syringyl lignin units; $\mathrm{pCa}$, p-coumaric acid; and $\mathrm{Fa}$, ferulic acid. Italicized mass fragments indicate base peaks. 


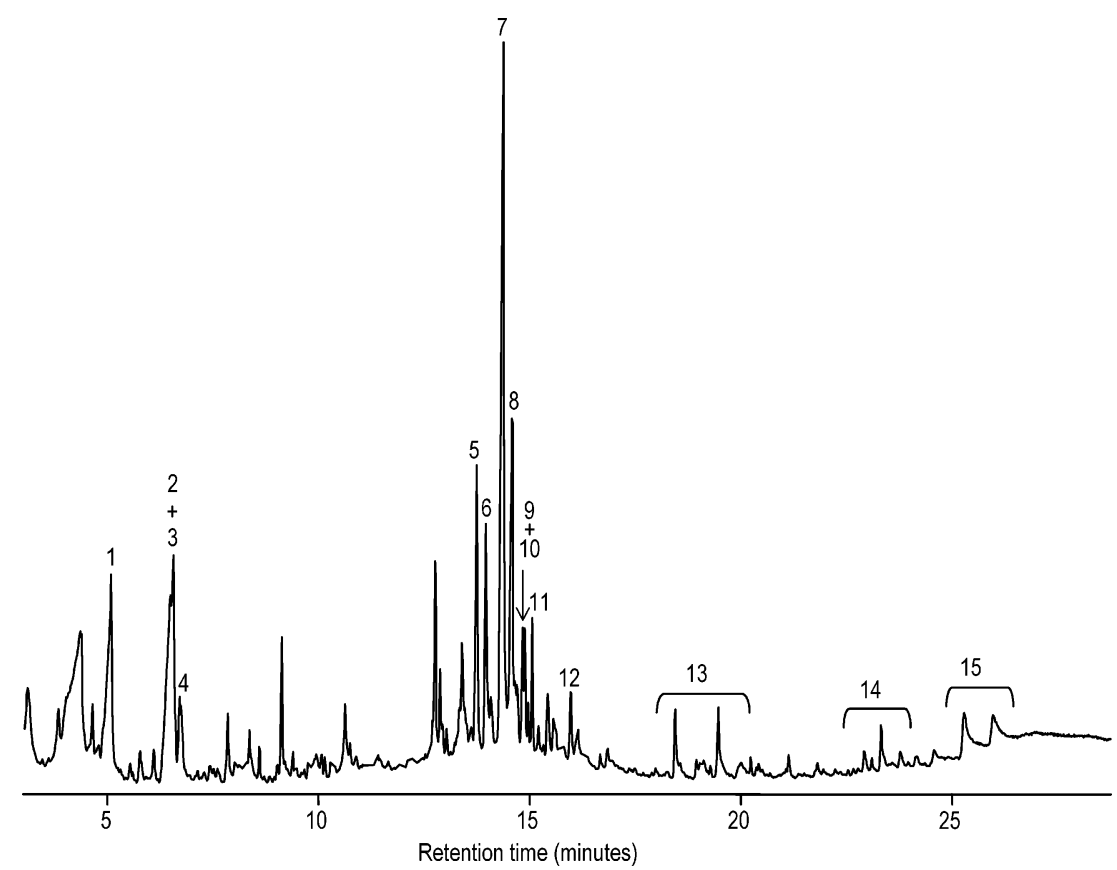

Figure 2. GC/MS chromatogram of the underivatized lipid extracts from abaca fibers. Labeling for the different peaks is as follows: 1, palmitic acid; 2 , linoleic acid; 3, oleic acid; 4, stearic acid; 5, campesterol; 6, stigmasterol; 7, sitosterol; 8, cycloartenone; 9, stigmasta-3,5-dien-7-one; 10, 24-methylenecycloartanone; 11, stigmast-4-en-3-one; 12, stigmastane-3,6-dione; 13, p-hydroxycinnamic acid esters; 14, sterol esters; and 15, triglycerides.

performed in splitless mode. Trimethylsilyl-diazomethane methylation and bis(trimethylsilyl)trifluoroacetamide (BSTFA) silylation, in the presence of pyridine, were used when required. Compounds were identified by comparing their mass spectra with mass spectra in the Wiley and NIST libraries and with those reported in the literature, by mass fragmentography and, when possible, by comparison with authentic standards.

Pyrolysis-GC/MS (Py-GC/MS). The pyrolysis of abaca fibers was performed in duplicate with a Curie point pyrolyzer (Horizon Instruments Ltd.) coupled to a Varian Saturn 2000 GC/MS, using a $30 \mathrm{~m} \times$ $0.25 \mathrm{~mm}$ i.d., $0.25 \mu \mathrm{m}$ DB-5 column. Approximately $100 \mu \mathrm{g}$ of finely divided sample was deposited on a ferromagnetic wire and then inserted into the glass liner and immediately placed in the pyrolyzer. The pyrolysis was carried out at $610{ }^{\circ} \mathrm{C}$. The chromatograph was programmed from $40(1 \mathrm{~min})$ to $300{ }^{\circ} \mathrm{C}$ at a rate of $6{ }^{\circ} \mathrm{C} / \mathrm{min}$. The final temperature was held for $20 \mathrm{~min}$. The injector, equipped with a liquid carbon dioxide cryogenic unit, was programmed from $-30(1 \mathrm{~min})$ to $300{ }^{\circ} \mathrm{C}$ at $200{ }^{\circ} \mathrm{C} / \mathrm{min}$, while the GC/MS interface was kept at $300{ }^{\circ} \mathrm{C}$. For the pyrolysis in the presence of tetramethylammonium hydroxide (TMAH), approximately $100 \mu \mathrm{g}$ of sample was mixed with $0.5 \mu \mathrm{L}$ of $25 \%$ TMAH. The wire was then inserted into the glass liner, which was subsequently placed in the pyrolyzer. The pyrolysis was carried out as described above. The compounds were identified by comparing the mass spectra obtained with those of the Wiley and NIST computer libraries and those reported in the literature $(23-25)$. Relative peak molar areas were calculated for carbohydrate and lignin pyrolysis products. The summed molar areas of the relevant peaks were normalized to $100 \%$, and the data for two repetitive pyrolysis experiments were averaged. The relative standard deviation for the pyrolysis data was less than $10 \%$. No attempt was made to calculate the response factor for every single compound released. However, for most of the lignin-derived phenols, the response factors were nearly identical (26), with the exception of vanillin, but this was a minor peak here.

\section{RESULTS AND DISCUSSION}

The abaca fiber is characterized by a relatively high lignin content (13.2\% of the total fiber). This value is higher than other nonwood bast fibers used for papermaking such as hemp or flax, with lignin content less than 5\% (27-29) but lower than wood (30). The content of lipids was about $0.4 \%$, similar to other nonwood materials used for papermaking such as flax or kenaf $(31,32)$ but lower than in woods. On the other hand, the hemicellulose fraction was mainly constituted by xylose, similarly to kenaf (32). Finally, the low content of the different metals, as shown in Table 1, supports the low ash content of the fiber $(0.5 \%)$.

Lignin Composition. To analyze in situ the chemical composition of lignin, the abaca fibers were subjected to Py-GC/ MS. The Py-GC/MS chromatogram is shown in Figure 1, and the identities and relative molar abundances of the released compounds are listed in Table 2. Carbohydrate pyrolysis products represented $52 \%$ on average, and phenols from lignin (and p-hydroxycinnamic acids) represented $48 \%$ of the total identified compounds from abaca fibers, in agreement with the relatively high lignin content of these fibers. The main compound identified in the pyrogram of abaca fiber was 4-vinylphenol ( $23 \%$ of the total released compounds) and generally considered as derived from $p$-hydroxyphenyl $(\mathrm{H})$ lignin moiety and/or $p$-coumaric acid. The extremely high content of 4-vinylphenol found after Py-GC/MS is somewhat surprising. However, most of the 4-vinylphenol released from abaca fibers is due to the presence of $p$-coumaric acid, which under the pyrolytic conditions decarboxylates to produce this compound (33). The presence of ferulic acid may also be biased since it will yield 4-vinylguaiacol as the main pyrolysis product, although 4-vinylguaiacol is only a very minor peak among the abaca pyrolysis products. Hydroxycinnamic acids, particularly $p$-coumaric and ferulic acids, occur widely in the cell walls of herbaceous plants forming cross-linkages between lignin and polysaccharides ( 9 , 34-39). The presence of these phenylpropanoid compounds constitutes a complication for lignin analysis by analytical pyrolysis since, as noted above, they yield products similar to those of corresponding lignin units. However, this problem can be solved by the use of pyrolysis in the presence of TMAH (Py/TMAH), which prevents decarboxylation and releases intact p-hydroxycinnamic acids (as their methyl derivatives) in addition to different lignin degradation products $(33,40-46)$.

$\mathrm{Py} / \mathrm{TMAH}$ of the abaca fibers released high amounts (over $60 \%$ of pyrolysis products) of the methyl derivative of $p$ - 
Table 3. Composition of Lipids (mg/100 g) from Abaca (M. textilis) Fibers ${ }^{a}$

\begin{tabular}{|c|c|c|c|c|}
\hline compounds & mass fragments & MW & formula & abaca \\
\hline $\begin{array}{l}\text { fatty acids } \\
n \text {-tetradecanoic acid } \\
\text { n-pentadecanoic acid } \\
\text { 9-hexadecenoic acid } \\
\text { n-hexadecanoic acid } \\
\text { n-heptadecanoic acid } \\
\text { 9,12-octadecadienoic acid } \\
\text { 9-octadecenoic acid } \\
\text { n-octadecanoic acid } \\
\text { n-nonadecanoic acid } \\
\text { n-eicosanoic acid } \\
\text { n-heneicosanoic acid } \\
\text { n-docosanoic acid } \\
\text { n-tricosanoic acid } \\
n \text {-tetracosanoic acid } \\
n \text {-pentacosanoic acid } \\
\text { n-hexacosanoic acid } \\
\text { n-heptacosanoic acid } \\
n \text {-octacosanoic acid } \\
\text { n-nonacosanoic acid } \\
n \text {-triacontanoic acid } \\
n \text {-dotriacontanoic } \\
n \text {-tetratriacontanoic acid } \\
n \text {-hexatriacontanoic acid }\end{array}$ & $\begin{array}{l}60 / 73 / 129 / 228 \\
60 / 73 / 129 / 242 \\
55 / 69 / 236 / 254 \\
60 / 73 / 129 / 256 \\
60 / 73 / 129 / 270 \\
67 / 81 / 280 \\
55 / 69 / 264 \\
60 / 73 / 129 / 284 \\
60 / 73 / 129 / 298 \\
60 / 73 / 129 / 312 \\
60 / 73 / 129 / 326 \\
60 / 73 / 129 / 340 \\
60 / 73 / 129 / 354 \\
60 / 73 / 129 / 368 \\
60 / 73 / 129 / 382 \\
60 / 73 / 129 / 396 \\
60 / 73 / 129 / 410 \\
60 / 73 / 129 / 424 \\
60 / 73 / 129 / 438 \\
60 / 73 / 129 / 452 \\
60 / 73 / 129 / 480 \\
60 / 73 / 129 / 508 \\
60 / 73 / 129 / 536\end{array}$ & $\begin{array}{l}228 \\
242 \\
254 \\
256 \\
270 \\
280 \\
282 \\
284 \\
298 \\
312 \\
326 \\
340 \\
354 \\
368 \\
382 \\
396 \\
410 \\
424 \\
438 \\
452 \\
480 \\
508 \\
536\end{array}$ & $\begin{array}{l}\mathrm{C}_{14} \mathrm{H}_{28} \mathrm{O} \\
\mathrm{C}_{15} \mathrm{H}_{30} \mathrm{O} \\
\mathrm{C}_{16} \mathrm{H}_{30} \mathrm{O} \\
\mathrm{C}_{16} \mathrm{H}_{32} \mathrm{O}_{2} \\
\mathrm{C}_{17} \mathrm{H}_{34} \mathrm{O}_{2} \\
\mathrm{C}_{18} \mathrm{H}_{32} \mathrm{O}_{2} \\
\mathrm{C}_{18} \mathrm{H}_{34} \mathrm{O}_{2} \\
\mathrm{C}_{18} \mathrm{H}_{36} \mathrm{O}_{2} \\
\mathrm{C}_{19} \mathrm{H}_{38} \mathrm{O}_{2} \\
\mathrm{C}_{20} \mathrm{H}_{40} \mathrm{O}_{2} \\
\mathrm{C}_{21} \mathrm{H}_{42} \mathrm{O}_{2} \\
\mathrm{C}_{22} \mathrm{H}_{44} \mathrm{O}_{2} \\
\mathrm{C}_{23} \mathrm{H}_{46} \mathrm{O}_{2} \\
\mathrm{C}_{24} \mathrm{H}_{48} \mathrm{O}_{2} \\
\mathrm{C}_{25} \mathrm{H}_{50} \mathrm{O}_{2} \\
\mathrm{C}_{26} \mathrm{H}_{52} \mathrm{O}_{2} \\
\mathrm{C}_{27} \mathrm{H}_{54} \mathrm{O}_{2} \\
\mathrm{C}_{28} \mathrm{H}_{56} \mathrm{O}_{2} \\
\mathrm{C}_{29} \mathrm{H}_{58} \mathrm{O}_{2} \\
\mathrm{C}_{30} \mathrm{H}_{60} \mathrm{O}_{2} \\
\mathrm{C}_{32} \mathrm{H}_{64} \mathrm{O}_{2} \\
\mathrm{C}_{34} \mathrm{H}_{68} \mathrm{O}_{2} \\
\mathrm{C}_{36} \mathrm{H}_{72} \mathrm{O}_{2}\end{array}$ & $\begin{array}{r}12.99 \\
0.12 \\
0.13 \\
0.25 \\
1.26 \\
0.17 \\
3.44 \\
3.93 \\
0.55 \\
0.08 \\
0.19 \\
0.10 \\
0.49 \\
0.26 \\
0.44 \\
0.40 \\
0.37 \\
0.20 \\
0.31 \\
0.10 \\
0.13 \\
0.04 \\
0.02 \\
0.01\end{array}$ \\
\hline $\begin{array}{l}\text { hydroxyfatty acids } \\
\alpha \text {-hydroxyfatty acids } \\
\text { 2-hydroxytetracosanoic acid } \\
\text { 2-hydroxypentacosanoic acid } \\
\omega \text {-hydroxyfatty acids } \\
\text { 22-hydroxydocosanoic acid } \\
\text { 24-hydroxytetracosanoic acid } \\
\text { 26-hydroxyhexacosanoic acid } \\
\text { 28-hydroxyoctacosanoic acid }\end{array}$ & $\begin{array}{l}73 / 411 / 455^{c} \\
73 / 425 / 469^{c} \\
\\
73 / 395 / 411 / 427^{c} \\
73 / 423 / 439 / 455^{c} \\
73 / 451 / 467 / 483^{c} \\
73 / 479 / 485 / 511^{c}\end{array}$ & $\begin{array}{l}470^{b} \\
484^{b} \\
442^{c} \\
470^{c} \\
498^{c} \\
526^{c}\end{array}$ & $\begin{array}{l}\mathrm{C}_{28} \mathrm{H}_{58} \mathrm{O}_{3} \mathrm{Sic}^{\mathrm{c}} \\
\mathrm{C}_{29} \mathrm{H}_{60} \mathrm{O}_{3} \mathrm{Si}^{\mathrm{c}} \\
\mathrm{C}_{26} \mathrm{H}_{54} \mathrm{O}_{3} \mathrm{Sic} \\
\mathrm{C}_{28} \mathrm{H}_{58} \mathrm{O}_{3} \mathrm{Sic}^{c} \\
\mathrm{C}_{30} \mathrm{H}_{62} \mathrm{O}_{3} \mathrm{Sic}^{c} \\
\mathrm{C}_{32} \mathrm{H}_{66} \mathrm{O}_{3} \mathrm{Si}^{c}\end{array}$ & $\begin{array}{l}0.82 \\
0.76 \\
0.06 \\
3.30 \\
0.62 \\
0.43 \\
1.13 \\
1.12\end{array}$ \\
\hline $\begin{array}{l}\text { fatty alcohols } \\
n \text {-docosanol } \\
n \text {-tetracosanol } \\
n \text {-hexacosanol } \\
n \text {-octacosanol } \\
n \text {-triacontanol }\end{array}$ & $\begin{array}{l}75 / 103 / 383^{b} \\
75 / 103 / 411^{b} \\
75 / 103 / 439^{b} \\
75 / 103 / 467^{b} \\
75 / 103 / 495^{b}\end{array}$ & $\begin{array}{l}398^{b} \\
426^{b} \\
454^{b} \\
482^{b} \\
510^{b}\end{array}$ & $\begin{array}{l}\mathrm{C}_{25} \mathrm{H}_{54} \mathrm{OSi}{ }^{b} \\
\mathrm{C}_{27} \mathrm{H}_{58} \mathrm{OSi}{ }^{b} \\
\mathrm{C}_{29} \mathrm{H}_{62} \mathrm{OSi} i^{b} \\
\mathrm{C}_{31} \mathrm{H}_{66} \mathrm{OSi}^{b} \\
\mathrm{C}_{33} \mathrm{H}_{70} \mathrm{OSi}^{b}\end{array}$ & $\begin{array}{l}2.22 \\
0.74 \\
0.40 \\
0.16 \\
0.55 \\
0.37\end{array}$ \\
\hline $\begin{array}{l}\text { steroid hydrocarbons } \\
\text { ergosta-3,5,7-triene } \\
\text { stigmasta-3,5,7-triene } \\
\text { stigmasta-3,5-diene }\end{array}$ & $\begin{array}{l}135 / 143 / 380 \\
135 / 143 / 394 \\
81 / 147 / 381 / 396\end{array}$ & $\begin{array}{l}380 \\
394 \\
396\end{array}$ & $\begin{array}{l}\mathrm{C}_{28} \mathrm{H}_{44} \\
\mathrm{C}_{29} \mathrm{H}_{46} \\
\mathrm{C}_{29} \mathrm{H}_{48}\end{array}$ & $\begin{array}{l}1.70 \\
0.35 \\
1.21 \\
0.14\end{array}$ \\
\hline $\begin{array}{l}\text { sterols } \\
\text { campesterol } \\
\text { stigmasterol } \\
\text { sitosterol } \\
\text { 7-oxocampesterol } \\
7 \text {-oxositosterol } \\
\text { cycloartenol } \\
\text { 24-methylenecycloartanol } \\
\text { cycloeucalenol } \\
\alpha \text {-tocoferol }\end{array}$ & $\begin{array}{l}55 / 145 / 213 / 382 / 400 \\
55 / 83 / 255 / 394 / 412 \\
145 / 213 / 396 / 414 \\
135 / 161 / 381 / 414 \\
135 / 161 / 395 / 428 \\
69 / 286 / 393 / 408 / 426 \\
95 / 300 / 407 / 422 / 440 \\
69 / 353 / 393 / 408 / 426 \\
165 / 205 / 430\end{array}$ & $\begin{array}{l}400 \\
412 \\
414 \\
414 \\
428 \\
426 \\
440 \\
426 \\
430\end{array}$ & $\begin{array}{l}\mathrm{C}_{28} \mathrm{H}_{48} \mathrm{O} \\
\mathrm{C}_{29} \mathrm{H}_{48} \mathrm{O} \\
\mathrm{C}_{29} \mathrm{H}_{50} \mathrm{O} \\
\mathrm{C}_{28} \mathrm{H}_{40} \mathrm{O}_{2} \\
\mathrm{C}_{29} \mathrm{H}_{42} \mathrm{O}_{2} \\
\mathrm{C}_{30} \mathrm{H}_{50} \mathrm{O} \\
\mathrm{C}_{31} \mathrm{H}_{52} \mathrm{O} \\
\mathrm{C}_{30} \mathrm{H}_{50} \mathrm{O} \\
\mathrm{C}_{29} \mathrm{H}_{50} \mathrm{O}_{2}\end{array}$ & $\begin{array}{r}13.45 \\
2.01 \\
2.20 \\
7.67 \\
0.05 \\
0.25 \\
0.54 \\
0.14 \\
0.59 \\
0.13\end{array}$ \\
\hline $\begin{array}{l}\text { steroid ketones } \\
\text { stigmasta-3,5-dien-7-ona } \\
\text { stigmast-4-en-3-ona } \\
\text { stigmast-4-en-3,6-diona } \\
\text { stigmastane-3,6-diona } \\
\text { cycloartenone } \\
\text { 24-methylenecycloartanone }\end{array}$ & $\begin{array}{l}174 / 269 / 410 \\
124 / 229 / 412 \\
137 / 398 / 408 / 411 / 426 \\
245 / 287 / 428 \\
95 / 381 / 409 / 424 \\
95 / 395 / 423 / 438\end{array}$ & $\begin{array}{l}410 \\
412 \\
426 \\
428 \\
424 \\
438\end{array}$ & $\begin{array}{l}\mathrm{C}_{29} \mathrm{H}_{50} \mathrm{O} \\
\mathrm{C}_{29} \mathrm{H}_{48} \mathrm{O} \\
\mathrm{C}_{29} \mathrm{H}_{46} \mathrm{O}_{2} \\
\mathrm{C}_{29} \mathrm{H}_{48} \mathrm{O}_{2} \\
\mathrm{C}_{30} \mathrm{H}_{48} \mathrm{O} \\
\mathrm{C}_{31} \mathrm{H}_{50} \mathrm{O}\end{array}$ & $\begin{array}{l}5.75 \\
1.13 \\
0.52 \\
0.06 \\
0.35 \\
3.02 \\
0.67\end{array}$ \\
\hline $\begin{array}{l}\text { steryl glycosides } \\
\text { campesteryl 3- } \beta \text {-D-glucopyranoside } \\
\text { stigmasteryl 3- } \beta \text {-D-glucopyranoside } \\
\text { sitosteryl 3- } \beta \text {-D-glucopyranoside }\end{array}$ & $\begin{array}{l}204 / 217 / 361 / 383^{b} \\
204 / 217 / 361 / 395^{b} \\
204 / 217 / 361 / 397^{b}\end{array}$ & $\begin{array}{l}850^{b} \\
862^{b} \\
864^{b}\end{array}$ & $\begin{array}{l}\mathrm{C}_{46} \mathrm{H}_{90} \mathrm{O}_{6} \mathrm{Si}_{4}^{b} \\
\mathrm{C}_{47} \mathrm{H}_{90} \mathrm{O}_{6} \mathrm{Si}_{4}^{b} \\
\mathrm{C}_{47} \mathrm{H}_{92} \mathrm{O}_{6} \mathrm{Si}_{4}^{b}\end{array}$ & $\begin{array}{l}1.48 \\
0.25 \\
0.08 \\
1.15\end{array}$ \\
\hline $\begin{array}{l}\text { sterol esters } \\
\text { campesterol esters } \\
\text { stigmasterol esters } \\
\text { sitosterol esters } \\
\text { cycloartenol esters } \\
24 \text {-methylenecycloartanol esters }\end{array}$ & $\begin{array}{l}147 / 255 / 367 / 382 \\
145 / 255 / 379 / 394 \\
147 / 255 / 381 / 396 \\
95 / 365 / 393 / 408 \\
95 / 379 / 407 / 422\end{array}$ & $\begin{array}{l}638 \S \\
650 \S \\
652^{\S} \\
664 \S \\
678 \S\end{array}$ & $\begin{array}{l}\mathrm{C}_{44} \mathrm{H}_{78} \mathrm{O}_{2} \S \\
\mathrm{C}_{45} \mathrm{H}_{78} \mathrm{O}_{2} \S \\
\mathrm{C}_{45} \mathrm{H}_{80} \mathrm{O}_{2} \S \\
\mathrm{C}_{46} \mathrm{H}_{80} \mathrm{O}_{2} \S \\
\mathrm{C}_{47} \mathrm{H}_{80} \mathrm{O}_{2} \S\end{array}$ & $\begin{array}{l}1.75 \\
0.06 \\
0.06 \\
0.59 \\
0.13 \\
0.91\end{array}$ \\
\hline $\begin{array}{l}\text { monoglycerides } \\
\text { 2,3-dihydroxypropyltetradecanoate } \\
\text { 2,3-dihydroxypropylhexadecanoate } \\
\text { 2,3-dihydroxypropyloctadec-9-enoate } \\
\text { 2,3-dihydroxypropyloctadecanoate } \\
\text { 2,3-dihydroxypropylhexacosanoate } \\
\text { 2,3-dihydroxypropylheptacosanoate } \\
\text { 2,3-dihydroxypropyloctacosanoate } \\
\text { 2,3-dihydroxypropylnonacosanoate } \\
\text { 2,3-dihydroxypropyltriacontanoate }\end{array}$ & $\begin{array}{l}73 / 147 / 203 / 343 / 431^{b} \\
73 / 147 / 203 / 371 / 459^{b} \\
73 / 129 / 147 / 397 / 485^{b} \\
73 / 147 / 203 / 399 / 487^{b} \\
73 / 147 / 203 / 511 / 599^{b} \\
73 / 147 / 203 / 525 / 613^{b} \\
73 / 147 / 203 / 539 / 627^{b} \\
73 / 147 / 203 / 553 / 641^{b} \\
73 / 147 / 203 / 567 / 655^{b}\end{array}$ & $\begin{array}{l}446^{b} \\
474^{b} \\
500^{b} \\
502^{b} \\
614^{b} \\
628^{b} \\
642^{b} \\
656^{b} \\
670^{b}\end{array}$ & $\begin{array}{l}\mathrm{C}_{23} \mathrm{H}_{50} \mathrm{O}_{4} \mathrm{Si}_{2}{ }^{b} \\
\mathrm{C}_{25} \mathrm{H}_{54} \mathrm{O}_{4} \mathrm{Si}_{2}{ }^{b} \\
\mathrm{C}_{27} \mathrm{H}_{56} \mathrm{O}_{4} \mathrm{Si}_{2}{ }^{b} \\
\mathrm{C}_{27} \mathrm{H}_{58} \mathrm{O}_{4} \mathrm{Si}_{2}{ }^{b} \\
\mathrm{C}_{35} \mathrm{H}_{74} \mathrm{O}_{4} \mathrm{Si}_{2}{ }^{b} \\
\mathrm{C}_{36} \mathrm{H}_{76} \mathrm{O}_{4} \mathrm{Si}_{2}{ }^{b} \\
\mathrm{C}_{37} \mathrm{H}_{78} \mathrm{O}_{4} \mathrm{Si}_{2}{ }^{b} \\
\mathrm{C}_{38} \mathrm{H}_{80} \mathrm{O}_{4} \mathrm{Si}_{2}{ }^{b} \\
\mathrm{C}_{39} \mathrm{H}_{82} \mathrm{O}_{4} \mathrm{Si}_{2}{ }^{b}\end{array}$ & $\begin{array}{l}3.05 \\
0.04 \\
0.23 \\
0.56 \\
0.22 \\
0.80 \\
0.21 \\
0.88 \\
0.05 \\
0.06\end{array}$ \\
\hline $\begin{array}{l}\text { diglycerides } \\
\text { 1,2-dipalmitoylglycerol } \\
\text { 1,3-dipalmitoylglycerol } \\
\text { 1-palmitoyl-2-stearoylglycerol + } \\
\text { 1-stearoyl-2-palmitoylglycerol }\end{array}$ & $\begin{array}{l}73 / 129 / 313 / 625^{b} \\
73 / 129 / 313 / 371 / 625^{b} \\
73 / 313 / 341 / 385 / 413^{b}\end{array}$ & $\begin{array}{l}640^{b} \\
640^{b} \\
668^{b}\end{array}$ & $\begin{array}{l}\mathrm{C}_{38} \mathrm{H}_{76} \mathrm{O}_{5} \mathrm{Si} \\
\mathrm{C}_{38} \mathrm{H}_{76} \mathrm{O}_{5} \mathrm{Si} \\
\mathrm{C}_{40} \mathrm{H}_{80} \mathrm{O}_{5} \mathrm{Si}\end{array}$ & $\begin{array}{l}1.70 \\
0.08 \\
0.15 \\
0.17\end{array}$ \\
\hline
\end{tabular}


Table 3. Continued

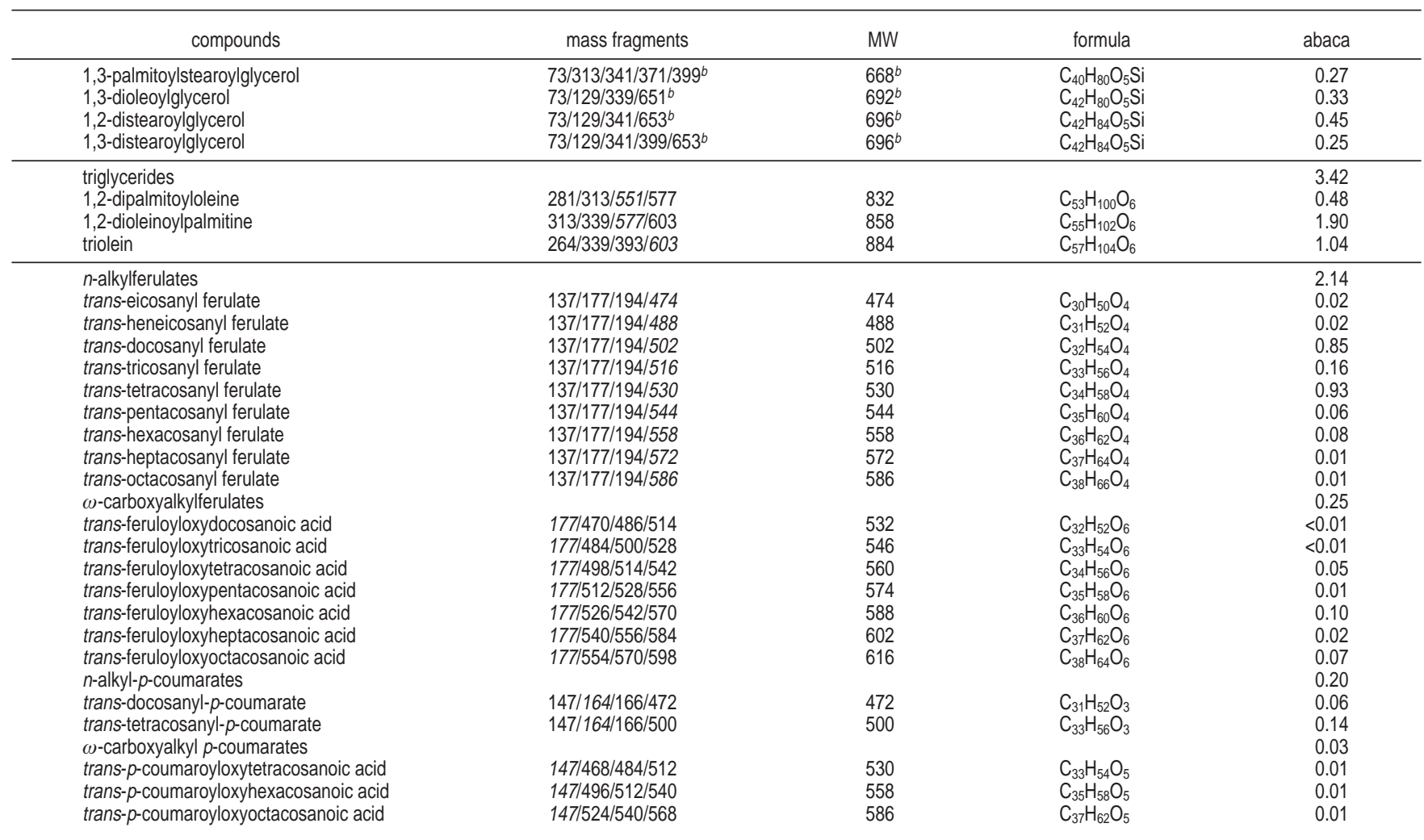

a Main mass fragments, molecular weight (MW), and formula are included. ${ }^{b}$ As TMS ether derivatives. ${ }^{c}$ As methyl ester and TMS ether derivatives. $\S$ only the molecular weight and formula of the main constituent of the series (i.e., palmitic acid ester) are shown. Italicized mass fragments indicate base peaks.

coumaric acid, i.e., 3-(4-methoxyphenyl)propenoic acid methyl ester, as well as lower amounts (2.5\% of pyrolysis products) of the methyl derivative of ferulic acid, i.e., 3-(3,4-dimethoxyphenyl)propenoic acid methyl ester. $p$-Hydroxycinnamic acids are present in abaca fiber in very high amounts as compared to lignin (cinnamic acids/lignin molar ratio of 2.64, estimated after Py/TMAH). This result agrees well with the high amounts of 4-vinylphenol released upon Py-GC/MS. Studies on maize (47), wheat (48), and other grasses, including bamboo (49), revealed that $p$-coumaric acid is esterified at the $\gamma$-position of lignin side chains and predominantly to $\mathrm{S}$ units $(49,50)$. Therefore, probably the major part of $p$-coumaric acid in abaca fiber cell walls may also be attached at the $\gamma$-position of the lignin side chain. Similar results have been reported by Sun et al. (9) that demonstrated that most $p$-coumaric acid in abaca fiber was esterified to lignin. Ferulic acid, on the other hand, although it is a minor component in abaca fiber, is mostly etherified to lignin (9). Therefore, the high content of $p$-hydroxycinnamic acids, particularly $p$-coumaric acid, in abaca fiber seems to be advantageous for pulping since ester bonds are easily cleaved during alkaline cooking.

The pyrogram of the abaca fiber also showed compounds derived from $p$-hydroxyphenyl $(\mathrm{H})$, guaiacyl $(\mathrm{G})$, and syringyl (S) lignin units, with a very strong predominance of the S-units. The main lignin-derived compounds identified were syringol (37), 4-methylsyringol (42), 4-vinylsyringol (49), and trans-4propenylsyringol (56). Syringaldehyde (53), trans-sinapaldehyde (67), and trans-sinapyl alcohol (68) were also identified. The $\mathrm{H}$ and $\mathrm{G}$ lignin counterparts were also detected although in lower amounts, and the H:G:S molar ratio accounted for 1.5:1:4.9. The high $\mathrm{S} / \mathrm{G}$ ratio observed in the lignin of abaca fibers upon Py-GC/MS (S/G molar ratio of 4.9) is typical of hardwoods and similar to other nonwoody plants such as kenaf, with a $S / G$ of 5.4 (32). However, other nonwood fibers, such as flax or hemp, have lower ratios $(\mathrm{S} / \mathrm{G}<1.0)$. The high $\mathrm{S}$-lignin content observed in the abaca fibers is advantageous for delignification during pulping because the $\mathrm{S}$ lignin is mainly linked by more labile ether bonds, is relatively unbranched, and has a lower condensation degree than the $\mathrm{G}$ lignin $(51,52)$. The higher reactivity of the $\mathrm{S}$-lignin with respect to the $\mathrm{G}$ lignin in alkaline systems is known (53). The G-units have a C-5 aromatic position available for very strong carbon-carbon bonds, which make them fairly resistant to the pulping depolymerization. Therefore, the high $\mathrm{S} / \mathrm{G}$ ratio of the lignin in abaca fibers makes it easier to be delignified because of the lower condensation degree of the lignin, despite having a higher lignin content than other fibers such as flax or hemp. In fact, the ease of delignification and bleaching of abaca pulp with respect to other fibers with lower $\mathrm{S} / \mathrm{G}$ ratio, such as hemp or flax, has been revealed by the pulp mill using these fibers (personal communication).

Finally, a detailed analysis of the compounds released after Py-GC/MS of abaca fibers revealed the presence of sinapyl acetate [3-(4-hydroxy-3,5-dimethoxyphenyl)-2-propen-1-ol acetate] in cis and trans forms (peaks 69 and 70, respectively). Minor amounts of coniferyl acetates in cis and trans forms (peaks 62 and 65, respectively) were also detected. Acetylated lignin units have already been reported to occur in kenaf (32, 54). Sinapyl and coniferyl acetates have also been detected upon $\mathrm{Py}-\mathrm{GC} / \mathrm{MS}$ in other nonwood fibers with a high $\mathrm{S} / \mathrm{G}$ ratio such as sisal and jute (25). The presence of sinapyl acetates in abaca fibers is shown in the inset of Figure 1. A predominance of the trans over the cis form was observed, similar to that of the respective nonacetylated sinapyl alcohols. Previous NMR and degradative studies $(54,55)$ have shown that lignin in kenaf is acetylated at the $\gamma$-position of the side chain and that this acetylation occurred predominantly on S-units in agreement with 


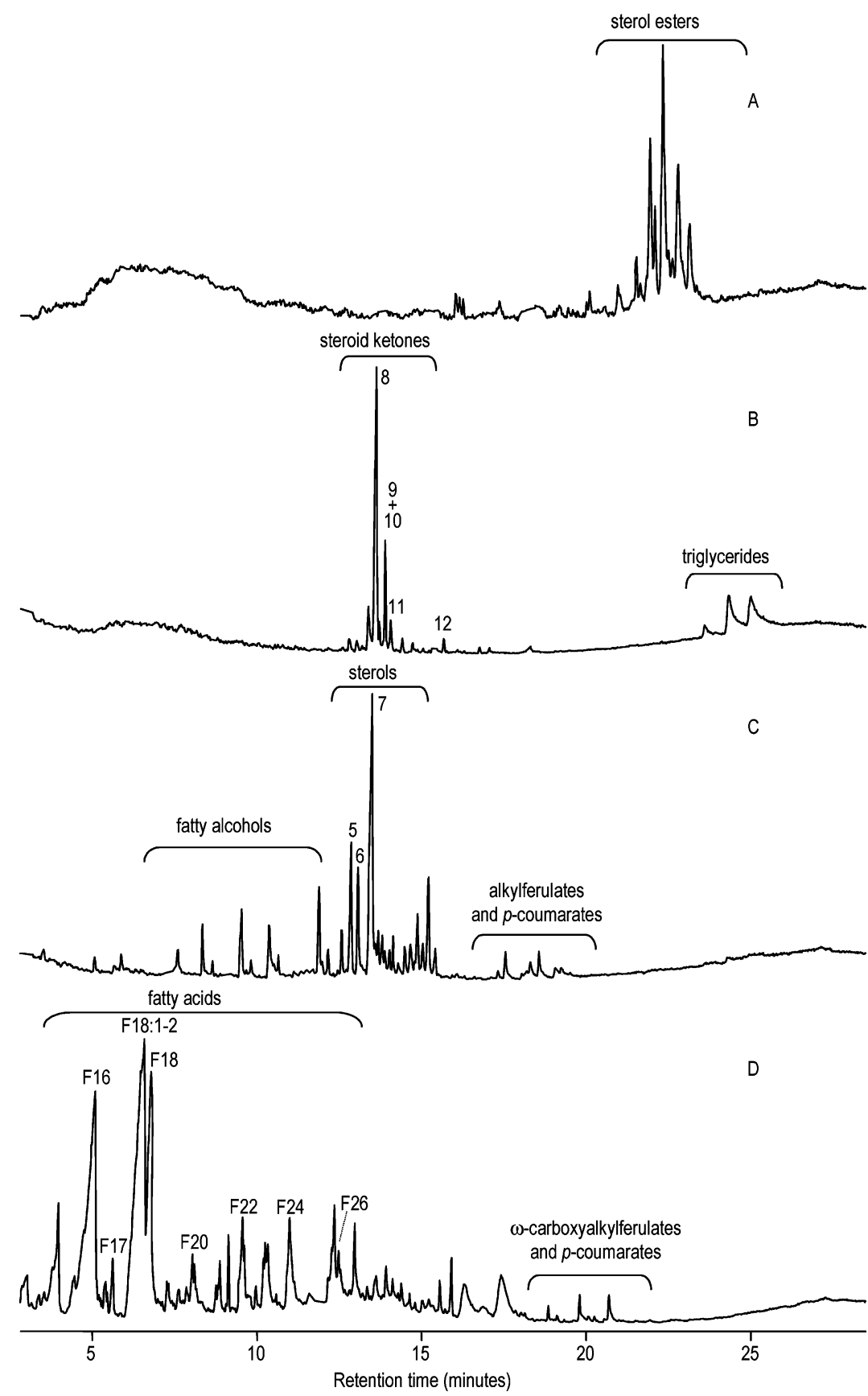

Figure 3. GC/MS chromatograms of the different SPE fractions isolated from the abaca fibers extracts. Fraction $\mathrm{A}$, eluted with $8 \mathrm{~mL}$ of hexane; fraction $B$, eluted with $6 \mathrm{~mL}$ of hexane:chloroform (5:1); fraction $\mathrm{C}$, eluted with $10 \mathrm{~mL}$ of chloroform; and fraction $\mathrm{D}$, eluted with $10 \mathrm{~mL}$ of diethyl ether:acetic acid (98:2). Labeling for the different peaks is as follows: 5, campesterol; 6, stigmasterol; 7, sitosterol; 8, cycloartenone; 9, stigmasta-3,5-dien-7-one; 10, 24-methylenecycloartanone; 11, stigmast-4-en-3-one; and 12, stigmastane-3,6-dione.

the present Py-GC/MS results. Moreover, it has recently been demonstrated that sinapyl acetate is a true lignin precursor in kenaf (56) involved in the polymerization of lignin along with the normal coniferyl and sinapyl alcohols. Therefore, it is also possible that sinapyl and coniferyl alcohol acetates are also lignin precursos in the case of abaca lignin.

Lipid Composition. The lipid extracts from abaca fibers accounted for $0.4 \%$ of total fibers. The underivatized, methylated, and/or silylated extracts were analyzed by GC and GC/ MS using short and medium length high temperature capillary columns, respectively, with thin films, according to the method previously described (22). The GC/MS chromatogram of the underivatized lipid extract from abaca fibers is shown in Figure 2, and the identities and abundances of the main lipophilic compounds are detailed in Table 3. For a better characteriza- tion of the compounds present in the total extracts of the abaca fibers, these extracts were subjected to SPE fractionation according to the method developed by Gutiérrez et al. (22). The chromatograms of the different SPE fractions are shown in Figure 3.

The most predominant lipids in abaca fibers were fatty acids (24\% of total extract) and free sterols (24\% of total extract). Additionally, significant amounts of steroid ketones $(10 \%)$, triglycerides $(6 \%), \omega$-hydroxyfatty acids $(6 \%)$, monoglycerides (4\%), fatty alcohols (4\%), and a series of $p$-hydroxycinnamyl ( $p$-coumaric and ferulic acids) esters were also found, as well as minor amounts of steroid hydrocarbons, diglycerides, $\alpha$-hydroxyfatty acids, sterol esters, and sterol glycosides. Among these compounds, it is interesting to note the identification of a series of $p$-coumaric and ferulic acids esterified with long chain 

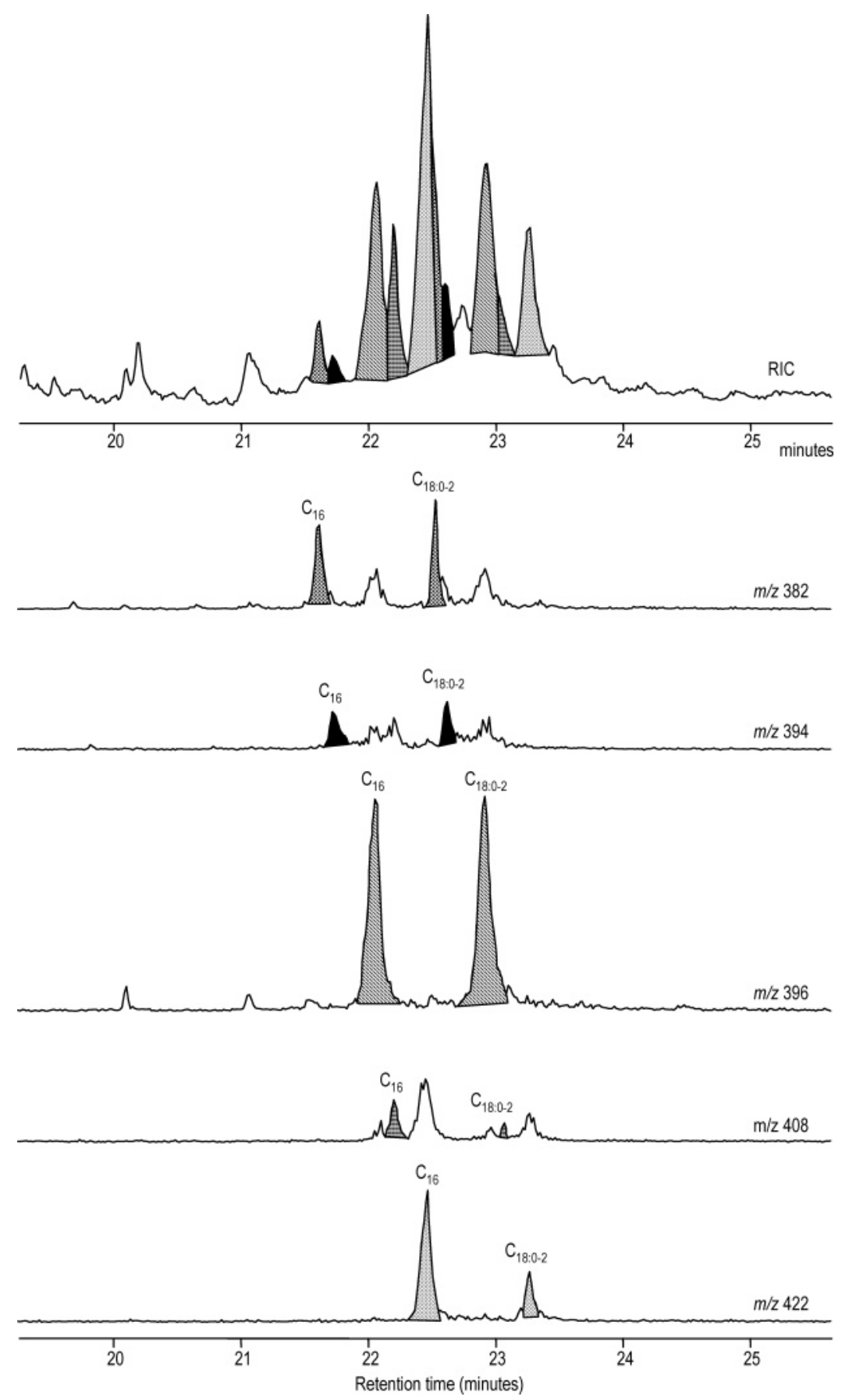

Figure 4. Single ion chromatograms showing the distribution of the different sterol esters in the abaca fiber (fraction A of the SPE). $m / z$ 382, campesterol esters; $\mathrm{m} / \mathrm{z}$ 394, stigmasterol esters; $\mathrm{m} / \mathrm{z}$ 396, sitosterol esters; $\mathrm{m} / \mathrm{z} 408$, cycloartenol esters; and $\mathrm{m} / \mathrm{z}$ 422, 24-methylenecycloartanol esters.

fatty alcohols and $\omega$-hydroxyfatty acids. The presence of theseseries of compounds in abaca has recently been reported (10) being the first time that the series of $p$-coumaric acid esterified with $\omega$-hydroxyfatty acids has been described in plants.

Sitosterol was the most important compound identified in the abaca extracts, together with other free sterols such as campesterol, stigmasterol, cycloeucalenol, cycloartenol, and 24-methylenecycloartanol. Most of these sterols are also present in other Musa species, such as Musa sapientum (57) and Musa paradisiaca (58). Some of these sterols were also found esterified with long chain fatty acids, and their structures were determined by their mass spectra. Figure 4 shows the distribution of the different esterified sterol esters by monitoring the characteristic fragments of the different sterol moieties in their mass spectra.
The sterol ester series corresponded to campesterol $(\mathrm{m} / \mathrm{z}, 382)$, stigmasterol ( $\mathrm{m} / \mathrm{z}, 394)$, sitosterol $(\mathrm{m} / \mathrm{z} 396)$, cycloartenol $(\mathrm{m} / \mathrm{z}$ 408), and 24-methylenecycloartanol $(\mathrm{m} / \mathrm{z} 422)$. All of the esterified sterol ester series showed two major peaks for the $\mathrm{C}_{16}$ and $\mathrm{C}_{18}$ fatty acids. However, sterol esters do not show the molecular ion and only the sterol moiety can be determined by mass spectrometry. For a complete characterization of the sterol esters, the SPE fraction enriched in sterol esters (shown in Figure 3) was further subjected to saponification. Thus, it could be possible to determine the composition of the sterols and fatty acids that were esterified in the abaca extracts. The composition of the fatty acids and sterols released after saponification of the sterol ester-enriched fraction of the abaca extracts is shown in Table 4. The esterified fatty acids ranged from $\mathrm{C}_{14}$ to $\mathrm{C}_{28}$, with maxima for the palmitic, linoleic, oleic, and stearic acids. 
Table 4. Composition and Relative Abundance (\%) of the Fatty Acids and Sterols Released after Saponification of the Sterol Ester-Enriched Fraction of the Abaca Extracts

\begin{tabular}{lrll}
\hline \multicolumn{1}{c}{ compounds } & $\%$ & \multicolumn{1}{c}{ compounds } & $\%$ \\
\hline & \multicolumn{2}{c}{ fatty acids } & \\
n-tetradecanoic acid & 6 & $n$-heneicosanoic acid & 0.2 \\
$n$-pentadecanoic acid & 5 & $n$-docosanoic acid & 2 \\
$n$-hexadecanoic acid & 29 & $n$-tricosanoic acid & 1 \\
$n$-heptadecanoic acid & 3 & $n$-tetracosanoic acid & 2 \\
9,12 -octadecadienoic acid & 9 & $n$-pentacosanoic acid & 1 \\
9-octadecenoic acid & 10 & $n$-hexacosanoic acid & 1 \\
$n$-octadecanoic acid & 28 & $n$-heptacosanoic acid & 0.2 \\
$n$-nonadecanoic acid & 1 & $n$-octacosanoic acid & 0.4 \\
$n$-eicosanoic acid & 2 & & \\
& & sterols & 10 \\
campesterol & 16 & cycloartenol & 30 \\
stigmasterol & 16 & 24-methylenecycloartanol & \\
sitosterol & 34 & & \\
\hline
\end{tabular}

Among the esterified sterols identified are sitosterol, stigmasterol, campesterol, 24-methylenecycloartanol, and cycloartenol, the most abundant being sitosterol and 24-methylenecycloartanol.

On the other hand, several steroid ketones (such as stigmast4-en-3-one, stigmasta-3,5-dien-7-one, stigmast-4-en-3,6-dione, stigmastane-3,6-dione, cycloartenone, and 24-methylenecycloartanone, with cycloartenone being especially abundant) and steroid hydrocarbons (such as ergosta-3,5,7-triene, stigmasta3,5-diene, and stigmasta-3,5,7-triene) were identified. Minor amounts of sterol glycosides, such as campesteryl, stigmasteryl, and sitosteryl $3 \beta$-D-glucopyranosides, were also identified in abaca fibers, with the latter being the most predominant. The identification of sterol glycosides was accomplished (after BSTFA derivatization of the lipid extract) by comparison with the mass spectra and relative retention times of authentic standards (59).

Free fatty acids were also abundant in the abaca fibers and were identified in the range from $\mathrm{C}_{14}$ to $\mathrm{C}_{36}$, with a strong evenover-odd carbon atom number predominance. The series was dominated by the unsaturated oleic $\left(\mathrm{C}_{18: 1}\right)$ and linoleic $\left(\mathrm{C}_{18: 2}\right)$ acids. A series of $n$-fatty alcohols (from $\mathrm{C}_{22}$ to $\mathrm{C}_{30}$ ) were also identified in the abaca fibers, although in lower amounts, with the presence of only the even carbon atom number homologues and docosanol $\left(\mathrm{C}_{22}\right)$ being the most abundant.

Series of $p$-hydroxycinnamate esters consisting of ferulic and $p$-coumaric acids esterified to long chain fatty alcohols $\left(\mathrm{C}_{20}-\right.$ $\left.\mathrm{C}_{28}\right)$ and $\omega$-hydroxyfatty acids $\left(\mathrm{C}_{22}-\mathrm{C}_{28}\right)$ were also identified among the extracts of the abaca fiber. The characterization of the individual compounds was achieved based on the mass spectra obtained by GC/MS of the underivatized and their methyl and/or TMS ether derivatives (10). The series of $n$-alkyl trans-ferulates occurred in the range from $\mathrm{C}_{20}$ to $\mathrm{C}_{28}$, with the presence of both the even and the odd carbon atom number homologues, $\mathrm{C}_{22}$ and $\mathrm{C}_{24}$, being the most prominent, as also occurs in other plant species (60). n-Alkylferulates with odd carbon number atoms have rarely been reported (61). A series of $n$-alkyl trans-p-coumarates could also be detected, although in minor amounts. This series included only the $\mathrm{C}_{22}$ and $\mathrm{C}_{24}$ alkyl moieties, with the latter being the most prominent. The series of $n$-alkyl ferulates and $p$-coumarates identified occurred mostly in the trans form, although some minor amounts of the cis isomer were also observed at lower retention times.

On the other hand, ferulic and $p$-coumaric acids were also found esterified with the hydroxyl group of $\omega$-hydroxyfatty acids. The feruloyl ester-linked $\omega$-hydroxyfatty acids identified ranged from $\mathrm{C}_{22}$ to $\mathrm{C}_{28}$, the $\mathrm{C}_{24}, \mathrm{C}_{26}$, and $\mathrm{C}_{28}$ homologues being the most abundant. The odd members of the series were also present but in minor amounts. Feruloyl esters of $\omega$-hydroxyfatty acids have rarely been reported in plants. A series of acidic esters derived from $\mathrm{C}_{22}$ to $\mathrm{C}_{29} \omega$-hydroxy fatty acids and transand $c i s$-ferulic acids was reported for the first time in Virola species (62). Small amounts of feruloyoxydocosanoic acid and feruloyloxyhexacosanoic acid were also recently reported in Eucalyptus globulus wood (63). Finally, a series of p-coumaroyl esters of $\omega$-hydroxyfatty acids could also be identified in minor amounts in abaca leaf fibers. This series was present in the range from $\mathrm{C}_{24}$ to $\mathrm{C}_{28}$, with only the presence of the even carbon atom members.

Triglycerides (triolein, 1,2-dioleioylpalmitin, and 1,2-dipalmitoylolein) were also present in the abaca fibers, in contrast to other nonwood fibers such as flax and kenaf, which lack triglycerides $(31,32)$. Mono- and diglycerides were also present although in lower amounts. Monoglycerides were found in the range from $\mathrm{C}_{14}$ to $\mathrm{C}_{30}$, with $\mathrm{C}_{28}$ and $\mathrm{C}_{26}$ being the most abundant and with the exclusive presence of the isomer in position 1 . Diglycerides were also found, 1,2-distearin and 1,3-diolein being the most abundant.

The different lipid classes will have different behaviors during cooking. The sterol esters will only be partially hydrolyzed during alkaline cooking, while fatty acids can be extensively dissolved. At sufficiently high $\mathrm{pH}$ (as in alkaline pulping), the acids dissociate and form fatty acid soaps and can thus dissolve in water to quite a high extent, forming fatty acid soaps. On the other hand, sterols and fatty alcohols, steroid hydrocarbons and ketones, and steryl glycosides survive cooking. These compounds have a very low solubility in water and are difficult to remove and, therefore, can be at the origin of pitch problems.

In conclusion, the present work reports the chemical composition of lignins and lipids of the leaf fibers of abaca used for paper pulp manufacturing. The knowledge of chemical composition of main components of abaca fibers will be useful for a better utilization of nonwood plants.

\section{ACKNOWLEDGMENT}

We thank Isabel M. Rodríguez for sample preparation and technical support. We also thank J. M. Gras and G. Artal (CELESA, Spain) for providing the abaca leaf fibers.

\section{LITERATURE CITED}

(1) Peralta, A. G. Pulp produced from decorticated abaca fiber. Tappi J. 1996, 79, 263-266.

(2) Mita, A.; Kashiwabnara, S.; Pono, R. Q. Preparation of hydrogen peroxide alkaline pulp (PAP) from abaca. Pulping Conference; TAPPI Press: Atlanta, 1988.

(3) Estudillo, C. P.; Torres, A. S.; Austria, C. O. Production of abaca mechanical pulp as extender to waste paper for printing and writing paper. FPRDI J. 1998, 24, 11-24.

(4) Mabilangan, L. C.; Torres, A. S.; Mari, E. L.; Estudillo, C. P. Production of base paper for currency notes. FPRDI J. 1998, 24, 1-9.

(5) Yu, Y. Z.; Kettunen, H.; Hiltunen, E. J.; Niskanen, K. J. Comparison of abaca and spruce as reinforcement fibre. APPITA J. 2000, 53, 287-291.

(6) Morimoto, M. Nonwood plant resources. The status quo and their feasibility to paper industry. Tappi J. 2001, 55, 49-63.

(7) Jiménez, L.; Ramos, E.; Rodríguez, A.; de la Torre, M. J.; Ferrer, J. L. Optimization of pulping conditions of abaca. An alternative raw material for producing cellulose pulp. Biores. Technol. 2005, 96, 977-983. 
(8) Sun, R. C.; Fang, J. M.; Goodwin, A.; Lawther, J. M.; Bolton, $\mathrm{J}$. Isolation and characterization of polysaccharides from abaca fibre. J. Agric. Food Chem. 1998, 46, 2817-2822.

(9) Sun, R. C.; Fang, J. M.; Goodwin, A.; Lawther, J. M.; Bolton, $\mathrm{J}$. Fractionation and characterization of ball-milled and enzyme lignins from abaca fibre. J. Sci. Food Agric. 1999, 79, 10911098.

(10) del Río, J. C.; Rodríguez, M. J.; Gutiérrez, A. Identification of intact long-chain p-hydroxycinnamate esters in leaf fibers of abaca (Musa textilis) using gas chromatography/mass spectrometry. Rapid Commun. Mass Spectrom. 2004, 18, 2691-2696.

(11) González-Vila, F. J.; Almendros, G.; del Río, J. C.; Martín, F.; Gutiérrez, A.; Romero, J. Ease of delignification assessment of wood from different Eucalyptus species by pyrolysis (TMAH)GC/MS and CP/MAS ${ }^{13} \mathrm{C}$ NMR spectrometry. J. Anal. Appl. Pyrolysis 1999, 49, 295-305.

(12) del Río, J. C.; Gutiérrez, A.; Hernando, M.; Landín, P.; Romero, J.; Martínez, A. T. Determining the influence of eucalypt lignin composition in paper pulp yield using Py-GC/MS. J. Anal. Appl. Pyrolysis 2005, 74, 110-115.

(13) Hillis, W. E.; Sumimoto, M. Effect of extractives on pulping. In Natural Products of Woody Plants II; Rowe, J. W., Ed.; Springer-Verlag: Berlin, 1989; pp 880-920.

(14) del Río, J. C.; Gutiérrez, A.; González-Vila, F. J.; Martín, F.; Romero, J. Characterization of organic deposits produced in the kraft pulping of Eucalyptus globulus wood. J. Chromatogr. A 1998, 823, 457-465.

(15) Back, E. L.; Allen, L. H. Pitch Control, Wood Resin and Deresination; Tappi Press: Atlanta, 2000.

(16) Ali, M.; Sreekrishnan, T. R. Aquatic toxicity from pulp and paper mill effluents: A review. Adv. Environ. Res. 2001, 5, 175-196.

(17) Rigol, A.; LaTorre, A.; Lacorte, S.; Barceló, D. Bioluminiscence inhibition assays for toxicity screening of wood extractives and biocides in paper mill process waters. Environ. Toxicol. Chem. 2003, 23, 339-347.

(18) Technical Association of the Pulp and Paper Industry. Test Methods, 1992-1993; TAPPI: Atlanta, GA, 1993.

(19) Colinet, E.; Griepink, B.; Muntau, H. The certification of the contents of cadmium, copper, manganese, mercury, lead and zinc in two plant materials of aquatic origin (BCR numbers 60 and 61 ) and in olive leaves (BCR number 62). Commission of the European Communities BCR information-Reference materials. EUR 8119 EN; ECSC-EEG-EAEC: Brussels, Luxembourg, 1982.

(20) Griepink, B.; Muntau, H. The certification of the contents (mass fractions) of arsenic, cadmium, copper, lead, selenium and zinc in a sea lettuce (Ulva lactuca). BCR Information, Reference Materials; Commission of the European Communities: Brussels, 1987.

(21) Griepink, B.; Muntau, H. The certification of the contents (mass fractions) of As, B, Cd, Cu, Hg, Mn, Mo, Ni, Pb, Sb, Se and $\mathrm{Zn}$ in rye grass CRM 281, Commission of the European Communities, Report EUR 11839 EN, 1988.

(22) Gutiérrez, A.; del Río, J. C.; González-Vila, F. J.; Martín, F. Analysis of lipophilic extractives from wood and pitch deposits by solid-phase extraction and gas chromatography. J. Chromatogr. A 1998, 823, 449-455.

(23) Faix, O.; Meier, D.; Fortmann, I. Thermal degradation products of wood. A collection of electron-impact (EI) mass spectra of monomeric lignin derived products. Holz Roh-Werkst. 1990, 48, 351-354.

(24) Ralph, J.; Hatfield, R. D. Pyrolysis-GC/MS characterization of forage materials. J. Agric. Food Chem. 1991, 39, 1426-1437.

(25) del Río, J. C.; Gutiérrez, A.; Martínez, A. T. Identifying acetylated lignin units in nonwood fibers using pyrolysis-gas chromatography/mass spectrometry. Rapid Commun. Mass Spectrom. 2004, 18, 1181-1185.

(26) Bocchini, P.; Galletti, G. C.; Camarero, S.; Martínez, A. T. Absolute quantitation of lignin pyrolysis products using an internal standard. J. Chromatogr. A 1997, 773, 227-232.
(27) McDougall, G. J.; Morrison, I. M.; Stewart, D.; Weyers, J. D. B.; Hillman, J. R. Plant fibres: Botany, chemistry and processing for industrial use. J. Sci. Food Agric. 1993, 62, 1-20.

(28) Moore, G. Nonwood Fibre Applications in Papermaking; Pira International: Leatherhead, Surrey, United Kingdom, 1996.

(29) Lewin, M.; Pearce, E. M. Handbook of Fiber Chemistry; Marcel Dekker: New York, 1998.

(30) van Dam, J. E. G.; van Vilsteren, G. E. T.; Zomers, F. H. A.; Shannon, W. B.; Hamilton, I. T. Increased application of domestically produced plant fibres in textiles, pulp and paper production and composite materials. Directorate-General XII, Science, Research and Development; European Commission: Brussels, 1994

(31) Gutiérrez, A.; del Río, J. C. Lipids from flax fibers and their fate in alkaline pulping. J. Agric. Food Chem. 2003, 51, 49654971.

(32) Gutiérrez, A.; Rodríguez, I. M.; del Río, J. C. Chemical characterization of lignin and lipid fractions in kenaf bast fibers used for manufacturing high-quality papers. J. Agric. Food Chem. 2004, 52, 4764-4773.

(33) del Río, J. C.; Martín, F.; González-Vila, F. J. Thermally assisted hydrolysis and alkylation as a novel pyrolytic approach for the structural characterization of natural biopolymers and geomacromolecules. Trends Anal. Chem. 1996, 15, 70-79.

(34) Lam, T. B. T.; Kadoya, K.; Iiyama, K. Bonding of hydroxycinnamic acids to lignin: ferulic and $p$-coumaric acids are predominantly linked at the benzyl position of lignin, not the $\beta$-position, in grass cell walls. Phytochemistry 2001, 57, 987992.

(35) Sun, R. C.; Sun, X. F.; Zhang, S. H. Quantitative determination of hydroxycinnamic acids in wheat, rice, rye, and barley straws, maize stems, oil palm frond fiber, and fast-growing poplar wood. J. Agric. Food Chem. 2001, 49, 5122-5129.

(36) Grabber, J. H.; Ralph, J.; Hatfield, R. D. Cross-linking of maize walls by ferulate dimerization and incorporation into lignin. $J$. Agric. Food Chem. 2000, 48, 6106-6113.

(37) Scalbert, A.; Monties, B.; Lallemand, J.-Y.; Guittet, E.; Rolando, C. Ether linkage between phenolic acids and lignin fractions from wheat straw. Phytochemistry 1985, 24, 1359-1362.

(38) Sun, R. C.; Sun, X. F.; Wang, S. Q.; Zhu, W.; Wang, X. Y. Ester and ether linkages between hydroxycinnamic acids and lignins from wheat, rice, rye, and barley straws, maize stems, and fast-growing poplar wood. Ind. Crop Prod. 2002, 15, 179188.

(39) Lam, T. B. T.; Iiyama, K.; Stone, B. A. Cinnamic acid bridges between cell wall polymers in wheat and Phalaris internodes. Phytochemistry 1992, 31, 1179-1183.

(40) del Río, J. C.; McKinney, D. E.; Knicker, H.; Nanny, M. A.; Minard, R. D.; Hatcher, P. G. Structural characterization of bioand geo-macromolecules by off-line thermochemolysis with tetramethylammonium hydroxide. J. Chromatogr. A 1998, 823, 433-448.

(41) Martín, F.; del Río, J. C.; González-Vila, F. J.; Verdejo, T. Thermally assisted hydrolysis and alkylation of lignins in the presence of tetra-alkylammonium hydroxides. J. Anal. Appl. Pyrolysis 1995, 35, 1-13.

(42) Challinor, J. M. Characterization of wood by pyrolysis derivatisation-gas chromatography/mass spectrometry. J. Anal. Appl. Pyrolysis 1995, 35, 93-107.

(43) Clifford, D. J.; Carson, D. M.; MacKinney, D. E.; Bortiatynski, J. M.; Hatcher, P. G. A new rapid technique for the characterization of lignin in vascular plants: Thermochemolysis with tetramethylammonium hyroxide (TMAH). Org. Geochem. 1995, 23, 169-175.

(44) Kuroda, K.; Nishimura, N.; Izumi, A.; Dimmel, D. R. Pyrolysis of lignin in the presence of tetramethylammonium hydroxide: A convenient method for $\mathrm{S} / \mathrm{G}$ ratio determination. J. Agric. Food Chem. 2002, 50, 1022-1027. 
(45) Filley, T. R.; Minard, R. D.; Hatcher, P. G. Tetramethylammonium hydroxide (TMAH) thermochemolysis: Proposed mechanisms based upon the application of 13C-labeled TMAH to a synthetic model lignin dimer. Org. Geochem. 1999, 30, 607621.

(46) Vane, C. H. The molecular composition of lignin in spruce decayed by white-rot fungi (Phanerochaete chrysosporium and Trametes versicolor) using pyrolysis-GC-MS and thermochemolysis with tetramethylammonium hydroxide. Int. Biodeterior. Biodegrad. 2003, 51, 67-75.

(47) Ralph, J.; Hatfield, R. D.; Quideau, S.; Helm, R. F.; Grabber, J. H.; Jung, H.-J. G. Pathway of $p$-coumaric acid incorporation into maize lignin as revealed by NMR. J. Chem. Soc. 1994, 34, 112.

(48) Crestini, C.; Argyropoulos, D. S. Structural analysis of wheat straw lignin by quantitative ${ }^{31} \mathrm{P}$ and $2 \mathrm{D}$ NMR spectroscopy. The occurrence of ester bonds and $\alpha-\mathrm{O}-4$ substructures. J. Agric. Food Chem. 1997, 45, 1212-1219.

(49) Lu, F. C.; Ralph, J. Detection and determination of $p$-coumaroylated units in lignins. J. Agric. Food Chem. 1999, 47, 19881992.

(50) Grabber, J. H.; Quideau, S.; Ralph, J. p-Coumaroylated syringyl units in maize lignin: Implications for $\beta$-ether cleavage by thioacidolysis. Phytochemistry 1996, 43, 1189-1194.

(51) Nimz, H. Beech lignin- Proposal of a constitutional scheme. Angew. Chem. Int. Ed. 1974, 13, 313-321.

(52) Adler, E. Lignin chemistry-Past, present and future. Wood Sci. Technol. 1977, 11, 169-218.

(53) Tsutsumi, Y.; Kondo, R.; Sakai, K.; Imamura, H. The difference of reactivity between syringyl lignin and guaiacyl lignin in alkaline systems. Holzforschung 1995, 49, 423-428.

(54) Ralph, J. An unusual lignin from kenaf. J. Nat. Prod. 1996, 59, $341-342$
(55) Ralph, J.; Lu, F. The DFRC method for lignin analysis. A simple modification for identifying natural acetates on lignins. J. Agric. Food Chem. 1998, 46, 4616-4619.

(56) Lu, F.; Ralph, J. Preliminary evidence for sinapyl acetate as a lignin monomer in kenaf. Chem. Commun. 2002, 90-91.

(57) Knapp, F. F.; Nicholas, H. J. The distribution of sterols and steryl esters in the banana plant. Phytochemistry 1969, 8, 2091-2093.

(58) Banerji, N.; Sen, A. K.; Das, A. K. A new 9,19-cyclotriterpene from flowers of Musa paradisiaca (Banana). Indian J. Chem. 1982, 21B, 387-388.

(59) Gutiérrez, A.; del Río, J. C. Gas chromatography/mass spectrometry demonstration of steryl glycosides in eucalypt wood, Kraft pulp and process liquids. Rapid Commun. Mass Spectrom. 2001, 15, 2515-2520.

(60) Kolattukudy, P. E.; Espelié, K. E. Chemistry, biochemistry, and function of suberin and associated waxes. In Natural Products of Woody Plants; Rowe, J. W., Ed.; Springer-Verlag: Berlin, 1989; Vol. 1, pp 304-367.

(61) Baldé, A. M.; Claeys, M.; Pieters, L. A.; Wray, V.; Vlietinck, A. J. Ferulic acid esters from stem-bark of Pavetta owariensis. Phytochemistry 1991, 30, 1024-1026.

(62) Kawanishi, K.; Hashimoto, Y. Long chain esters of Virola species. Phytochemistry 1987, 26, 749-752.

(63) Freire, C. S. R.; Silvestre, A. J. D.; Neto, C. P. Identification of new hydroxy fatty acids and ferulic acid esters in the wood of Eucalyptus globulus. Holzforschung 2002, 56, 143-149.

Received for review December 2, 2005. Revised manuscript received March 22, 2006. Accepted March 31, 2006. This study was supported by the Spanish Ministerio de Ciencia y Tecnología (MCYT) (Projects AGL2002-00393 and AGL2005-01748).

JF053016N 